\title{
Numerical and Experimental Study on Stiffened Composite Panel Repaired by Bolted Joints under Compressive Load
}

\author{
Jifeng Xu, Yuanpei Lan, Xingming Zhang*, Kui Du \\ Beijing Key Laboratory of Civil Aircraft Structures and Composite Materials, Beijing Aeronautical Science \& Technology Research \\ Institute, Beijng, China \\ Email: *zhangxingming@comac.cc
}

How to cite this paper: Xu, J.F., Lan, Y.P., Zhang, X.M. and Du, K. (2018) Numerical and Experimental Study on Stiffened Composite Panel Repaired by Bolted Joints under Compressive Load. Journal of Applied Mathematics and Physics, 6, 1763-1771. https://doi.org/10.4236/jamp.2018.68151

Received: April 28, 2018

Accepted: August 28, 2018

Published: August 31, 2018

\begin{abstract}
Numerical and experimental study was conducted to investigate the failure mode and strength performance of stiffened composite panel repaired by bolted joints under compressive load, and the results were then compared with those from virgin stiffened composite panel without any damage. A finite element analysis model was established for repaired and virgin stiffened composite panels under compressive load, the 3D Hashin criteria was applied to identify the composite structure failure, and the secondary stress criteria was adopted to identify the adhesive failure between the base laminate and the stiffener. The failure modes of repaired stiffened composite panels were stiffened composite panels breaking off along the bolt joints. The experimental results were consistent with the finite element analysis results, indicating the reliability of the finite element analysis model.
\end{abstract}

\section{Keywords}

Stiffened Composite Panel, Bolted Joints Repair, Compression Performance, 3D Finite Element Model

\section{Introduction}

The application of composite material on civil aircraft indicates the advancement of design and manufacturing in aviation. With wide application of composite structures, structural damage repair technology becomes key point not only for safe operation of new generation civil aircraft, but also for sustainable market share, as in [1]. Bolted joints-repair is a frequently used method to recover original structural performance. Therefore, many researchers investigated properties 
and analysis methods of bolted joints-repaired composite structures under different loads in various aspects, as in [2].

Kassapoglou investigated the stress method for interfacial analysis of composite stiffened panels, as in [3]. Zhang studied the post-buckling behavior of repaired composite panels, as in [4]. Mahdi studied the mechanical performance of repaired stiffened panel, as in [5]. Nie studied tensile performances of bolted joints-repaired composite laminates, as in [2]. Zhao studied compression performance of composite stiffened panel by bolted joints-repair, as in [6]. Chen investigated the repair technique for aircraft composite panel with discrete-source damage, as in [7].

In this study, a 3D finite element model of stiffened composite panels under compressive load was established, and tests were performed on stiffened composite panels in virgin or repaired by bolted joints to investigate the failure mode and strength recovery ratio etc. Then the simulation results were compared with the experimental results to validate the fidelity of the finite element model.

\section{Experiment}

Rectangular composite laminates were used in the experiments. The lay-up of the stiffened composite panel is shown in Table 1, and the test specimen size is $410 \mathrm{~mm}$ by $310 \mathrm{~mm}$, with detailed dimension information shown in Figure 1. A hole was machined at the center of the stiffened composite panel to simulate penetrating damage, with the holes' diameters as $12 \mathrm{~mm}$ and $20 \mathrm{~mm}$, respectively. The damaged stiffened composite panel was repaired by bolted joints with a metal patch of Ti-6Al-4V.

The original stiffened composite panels were prepared with X850 composite material, and the patches were prepared with Ti-6Al-4V. Then the damaged stiffened composite panel and the patch were bolted jointed with HST10 hex-drive bolt. Two types of stiffened composite panels repaired by bolted joint were prepared, numbered JX-I-1, JX-I-2, JX-I-3 with $12 \mathrm{~mm}$ hole, and JX-II-1,

Table 1. Lay-up of the stiffened composite panel.

\begin{tabular}{ccc}
\hline Structure & Lay up & Thickness $(\mathrm{mm})$ \\
\hline Skin & {$[45 /-45 / 0 / 0 /-45 / 0 / 45 /-45 / 90]_{\mathrm{S}}$} & 3.42 \\
Web(C Type $)$ & {$[45 / 0 /-45 / 90 / 45 / 0]$} & 1.14 \\
Upper covering & {$[45 /-45 / 0 / 0 /-45 / 90 / 45 / 0]$} & 1.52 \\
Lower covering & {$[0 / 45 /-45 / 0]_{\mathrm{S}}$} & 1.52 \\
\hline
\end{tabular}

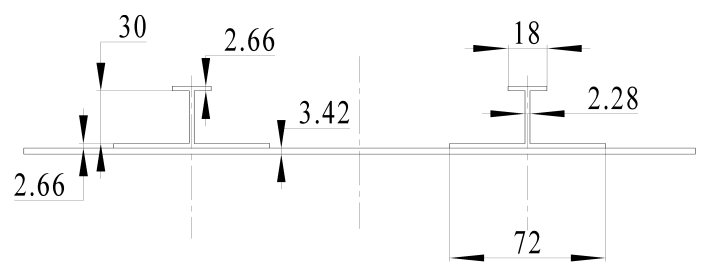

Figure 1. The stiffened composite panels. 
JX-II-2, JX-II-3 with $20 \mathrm{~mm}$ hole. Meanwhile, three virgin stiffened composite panels were prepared for comparison, numbered SP-1, SP-2, SP-3. The mechanical properties of X850 and Ti-6Al-4V are listed in Table 2, and the strength of X850 laminates are listed Table 3.

Strain gages were lapped on specimens to detect whether the load distribution was reasonable during the loading process, and the failure loads were identified and confirmed through the strain-load curves. There were over 100 strain gages arranged on each specimen, numbered S, F, X, M, and B for the upper flange, web, lower flange, skin, and the repaired region, respectively. In addition, the strain gages with numbers outside the parentheses were located on the stiffened side of the stiffened composite panels as shown in Figure 2.

Table 2. Mechanical properties of X850 laminates and Ti-6Al-4V.

\begin{tabular}{cccccccccc}
\hline X850 & $E_{11}(\mathrm{GPa})$ & $E_{22}(\mathrm{GPa})$ & $E_{33}(\mathrm{GPa})$ & $G_{12}(\mathrm{GPa})$ & $G_{23}(\mathrm{GPa})$ & $G_{13}(\mathrm{GPa})$ & $V_{12}$ & $V_{12}$ & $V_{12}$ \\
& 168.5 & 10.30 & 10.30 & 6.21 & 3.00 & 6.21 & 0.33 & 0.3 & 0.33 \\
& $E(\mathrm{GPa})$ & $V$ & & & & & & & \\
Ti-6Al-4V & & & & & & & & \\
& 110 & 0.3 & & & & & & & \\
\hline
\end{tabular}

Note: $E_{11}, E_{22}, E_{33}$-Young's moduli in the principal directions; $G_{12}, G_{23}, G_{13}$-shear moduli in the principal directions; $v_{12}, v_{23}, v_{13}$-Poisson's ratios in the principal directions; $E$-Young's modulus, $v$-Poisson's ratios.

Table 3. The strength of X850 laminates.

\begin{tabular}{ccccc}
\hline$X_{\mathrm{t}}(\mathrm{MPa})$ & $X_{\mathrm{c}}(\mathrm{MPa})$ & $Y_{\mathrm{t}}(\mathrm{MPa})$ & $Y_{\mathrm{C}}(\mathrm{MPa})$ & $S(\mathrm{GPa})$ \\
\hline 2785.6 & 1071.3 & 74.8 & 332.9 & 120.9 \\
\hline
\end{tabular}

Note: $X_{\mathrm{t}}-$ Tensile strength in 1 direction; $X_{\mathrm{c}}-$ Compressive strength in 1 direction; $Y_{\mathrm{t}}-$ Tensile strength in 2 direction; $Y_{\mathrm{c}}-$ Compressive strength in 2 direction; $S$ - shear strength.

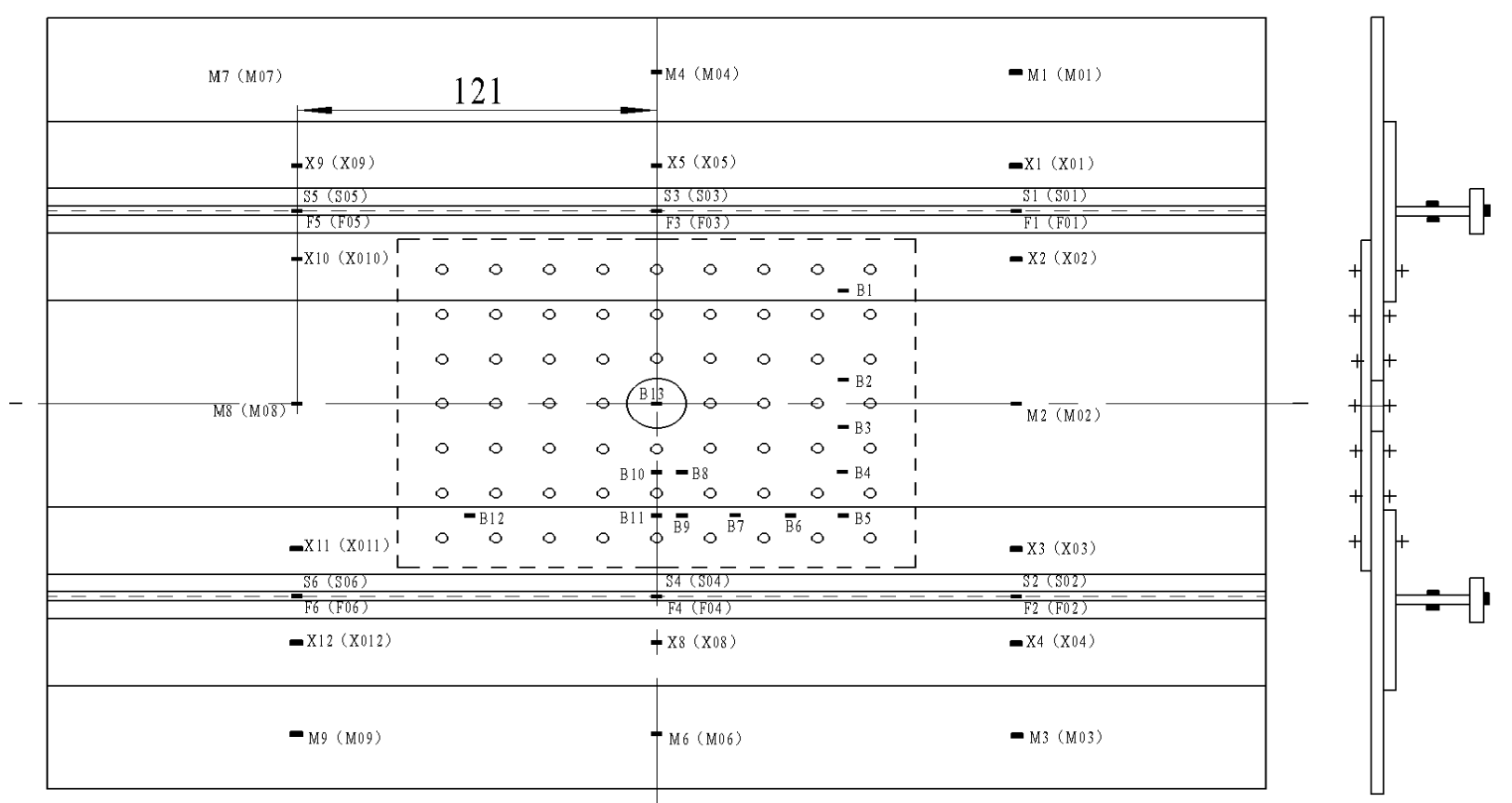

Figure 2. Distribution of strain gages on stiffened composite panels repaired by bolted joints under compressive load. 
Special fixture was developed for the experiments. Specimens were placed in the fixture to keep their edges in uniform and tight contact with the bottom face of the fixture. A griped stiffened composite panel for compressive load experiment is shown in Figure 3.

The specimens were loaded by stages, with a $1 \mathrm{~mm} / \mathrm{min}$ loading rate. The loading process was conducted in three stages. First, applying up to $15 \%$ of the estimated ultimate load and then the loading process was stopped to check whether the loading condition, connection of fixture with specimen and equipment, testing machine operation, strain measurement and data collection system were normal. Furthermore, specimens should not hold permanent deformation after unloading. Secondly, applying up to $15 \%$ of the estimated ultimate load on specimens again, ensuring relevant data measured was consistent with those from the first time loading and the strain value difference should be less than $10 \%$. Thirdly, applying up to $100 \%$ of the ultimate load, the initial and final failure load as well as failure mode, and correlation data were recorded.

\section{Finite Element Analysis}

A 3-D finite element model was established by ABAQUS with geometric parameters and material properties of the test specimens. Composite material was simulated with 3-D eight node hexahedron element (C3D8), and the element properties were allocated according to the properties of corresponding composite layers. The adhesive that bonded the stiffener and skin was simulated with cohesive element.

To simulate the actual boundary conditions of stiffened composite panels during experiments, one end of stiffened composite panel was fixed, and the other side was restricted by a coupling constraint with reference points. In addition, all

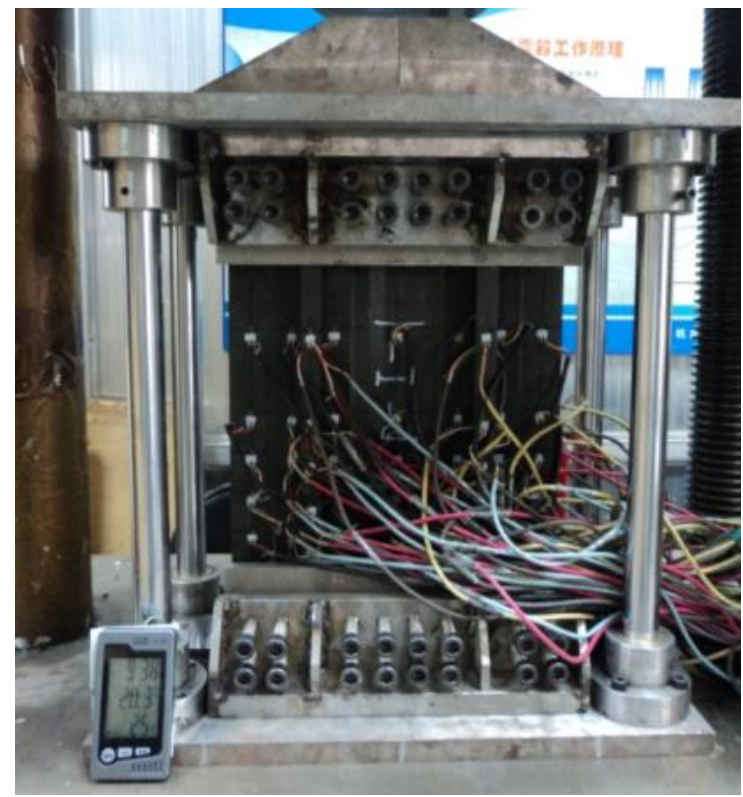

Figure 3. Griped stiffened composite panels for compressive load experiment. 
degrees of freedom for reference points were constrained despite of the loading direction. The displacement load was applied on reference points along the loading axis, and the displacement and force on reference points were recorded. Actual boundary conditions for stiffened composite panels in the finite element model are shown in Figure 4.

Since specimens' compressive instability was a complex nonlinear phenomenon, there existed a convergent issue with implicit solution method (Standard) in ABAQUS. To solve this problem, explicit solution method (Explicit) was applied to do quasi-static analysis during the loading process. Moreover, to simulate compressive specimens going through buckling process, buckle method was used to identify the instability modes first, and then imperfection method was used to introduce structure initial deformation in the form of minuscule instability mode.

Many failure criteria were established for composite laminate, such as stress-based, strain-based and energy-based failure criteria. To calculate efficiently without losing generality, damage of the laminates contact with the fixture was not taken into account. In this study, 3D Hashin criteria was applied to identify the damage in the skin laminate, the patch, and the stiffener, and the secondary stress criteria was adopted to identify the adhesive failure between the skin laminate and the stiffener.

\section{Results and Discussion}

\subsection{Failure Loads}

Failure loads on specimens of repaired and virgin stiffened composite panels under compressive load were analyzed, as shown in Table 4 . The failure loads of JX-I and JX-II are slightly higher than that of SP-I, and the stiffness of JX-I and JX-II are almost the same as SP-I (Figure 5). So the repaired stiffened composite panels had a high strength recovery rate and a high stiffness recovery rate. The

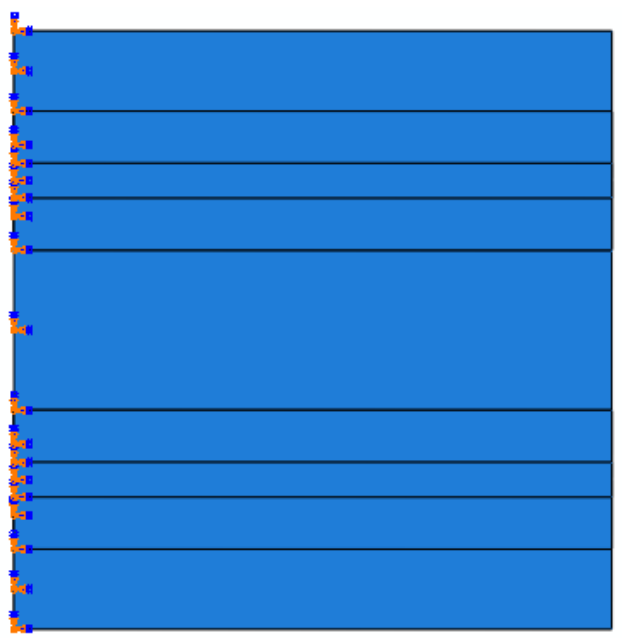

Figure 4. Boundary conditions of stiffened composite panels in the finite element model under compressive load. 
Table 4. Failure loads on specimens of repaired and virgin stiffened composite panels under compressive load.

\begin{tabular}{cccc}
\hline Specimen & SP-I & JX-I & JX-II \\
\hline Failure loads(kN) & 448 & 474 & 456 \\
\hline
\end{tabular}

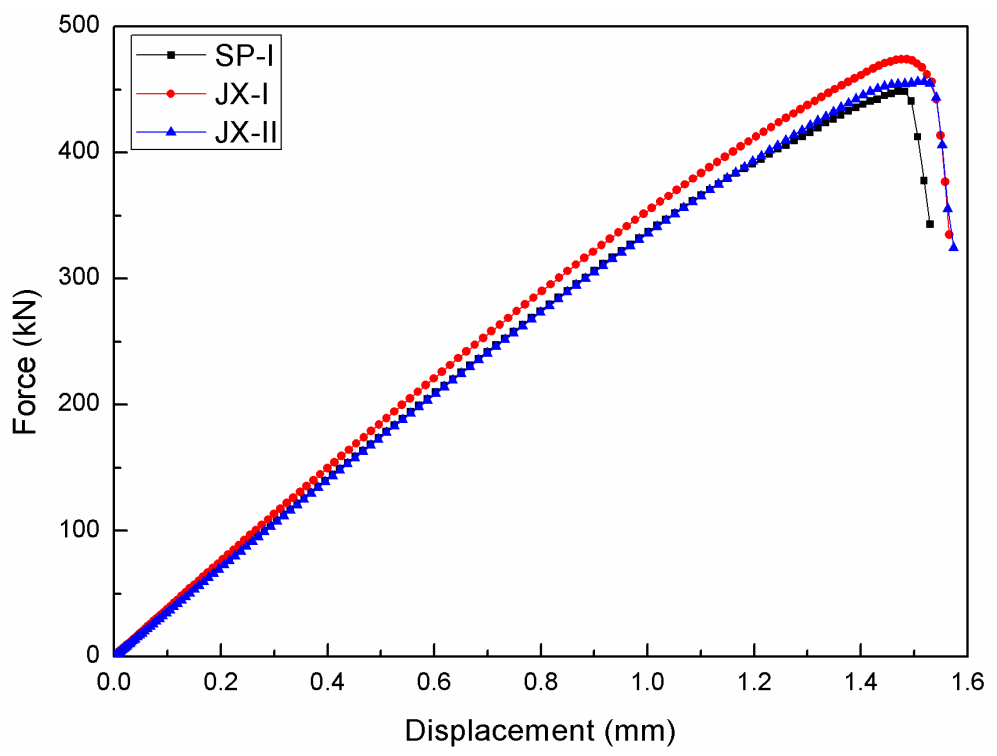

Figure 5. Relationship between the displacement and the load for SP-I, JX-I and JX-II.

experimental results were consistent with the finite element analysis results, despite of the failure loads of JX-I and JX-II were slight lower due to some manufacturing defects. In addition, the damage hole diameter had little effect on the failure loads in this test.

\subsection{Buckling Modes}

The analyzed buckling modes of SP-I were local buckling at non-stiffener region, namely skin buckling symmetrical by the central lines of the panel. A half wave emerged in the skin between the two stiffeners, and two reversed half waves emerged in the side skin (Figure 6).

The analyzed buckling modes of JX-I and JX-II were the same as SP-I at non-repaired region with two reversed half waves emerged in the side skin, while the skin buckling between the two stiffeners mostly located in the repaired region. The buckling loads were in the range of $200 \mathrm{kN}$ to $250 \mathrm{kN}$ for all the stiffened composite panels (Figure 6).

The actual load-stain curve of specimen JX-I-1 is shown in Figure 7. With increased compressive load, the specimen began to buckle locally, and the strain became bifurcate obviously on the compressive cross of the specimens. The bifurcation points on the load-strain curves of the specimens corresponding to the buckling loads. A Moire interferometry method was introduced to detect the stiffened composite panels buckling in compressive tests. The experimental results for SP-I were the same as those from the finite element analysis. But the 


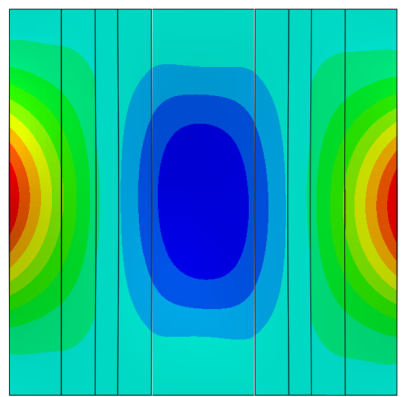

(a)

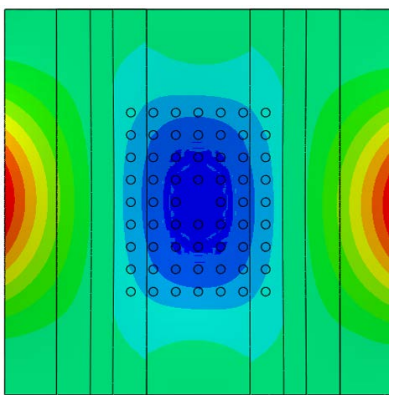

(b)

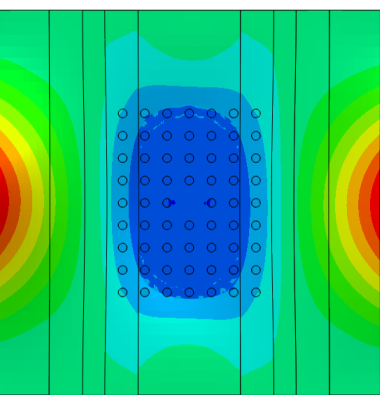

(c)

Figure 6. Buckling modes for SP-I, JX-I and JX-II. (a) SP-I; (b) JX-I; (c) JX-II.

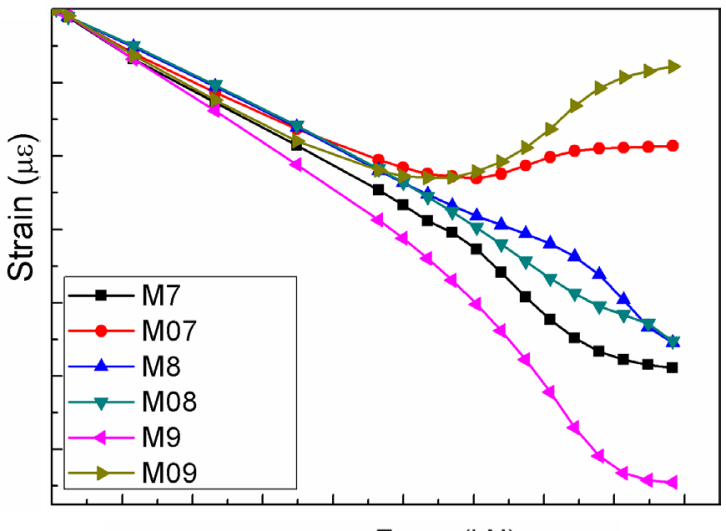

Force $(\mathrm{kN})$

Figure 7. Load-strain curves of JX-I-1 specimen.

skin buckling between the two stiffeners did not locate in the repaired region for JX-I and JX-II, due to the non-coplanar patch with the skin affecting the Moire pattern. Again the damage hole diameter had little effect on the buckling modes in this test.

\subsection{Failure Modes}

The stress distribution in the skin and stiffener for all stiffened composite panels were also analyzed. Stress concentration occurred in the middle, top and bottom of the specimens for SP-I. However, stress concentration happened at the damage hole and bolt edge for JX-I and JX-II. When the compressive load increasing near to the failure load, the fiber damage of stiffened composite panels occurred and expanded rapidly leading to the global load-carrying capability decrease, and finally the failure of the stiffened composite panels.

The failure modes of stiffened composite panels were corresponding to the buckling modes, and were consistent with the stress concentration distribution. The failure modes of SP-I were skin debonding in the middle of the stiffened composite panels as well as stiffeners breaking off when the load reached the failure loads. The failure modes of JX-I, JX-II were stiffened composite panels breaking off along the bolt joints (Figure 8). The experimental results were the same as the finite element analysis results, and the damage hole diameter had 


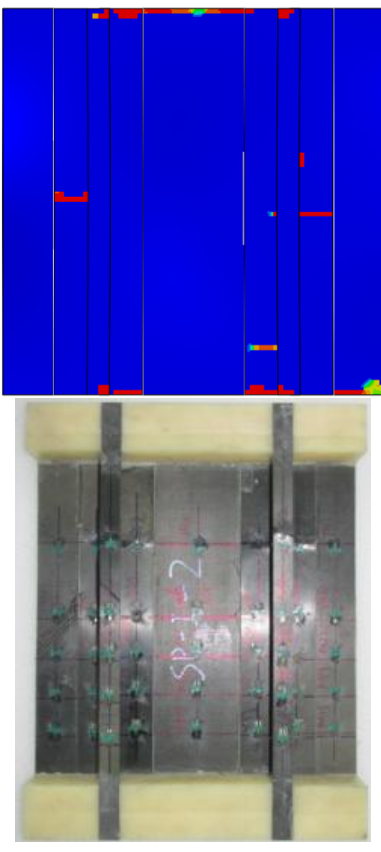

(a)
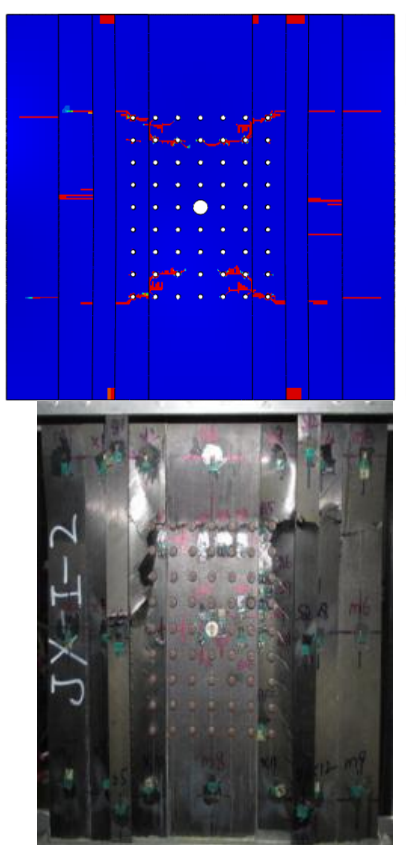

(b)

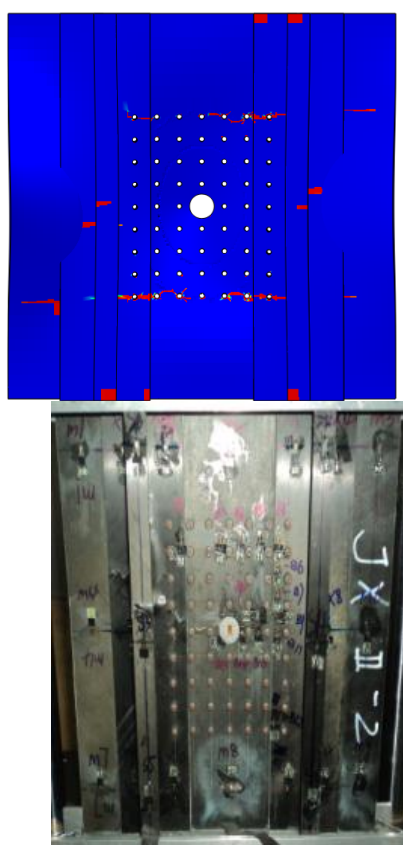

(c)

Figure 8. Failure modes for SP-I, JX-I and JX-II. (a) SP-I; (b) JX-I; (c) JX-II.

little effect on the failure modes in all test.

\section{Conclusion}

Experimental and numerical study indicated that the repaired stiffened composite panels had a desired recovery rate in both strength and stiffness. The buckling modes of repaired stiffened composite panels were the same as those of virgin stiffened composite panels at non-repaired region, while the skin buckling between the two stiffeners mostly located in the repaired region. The failure modes of repaired stiffened composite panels were panels breaking off along the bolt holes. The experimental results were consistent with the finite element analysis results, and the damage hole diameter had little effect on results in this study.

\section{References}

[1] Peng, J. and Shi, J. (2008) The Development Trend of Composite Materials for Large Aircraft Structure. Aeronautical. Science and Technology, 6, 3-5.

[2] Nie, H.C., Tan, R.M., Guo, X. and Guan, Z.D. (2016) Tensile Performances of Mechanically Fastened Repairs of Composite Laminates. Journal of Beijing University of Aeronautics and Astronautics, 42, 318-327.

[3] Kassapoglou, C. (1994) Stress Determination at Skin-Stiffener Interfaces of Composite Stiffened Panels under Generalized Loading. Journal of Reinforced Plastics and Composites, 13, 555-571. https://doi.org/10.1177/073168449401300605

[4] Zhang, H., Motipalli, J., Lam, Y.C. and Baker, A. (1998) Experimental and Finite Element Analyses on the Post-Buckling Behaviour of Repaired Composite Panels. Composites Part A: Applied Science and Manufacturing, 29, 1463-1471. https://doi.org/10.1016/S1359-835X(98)00030-X 
[5] Mahdi, S., Kinloch, A.J., Matthews, F.L. and Crisfield, M.A. (2002) The Mechanical Performance of repaired stiffened panels. Part II: Finite Element Modeling. Composites Part B: Engineering, 33, 355-366.

https://doi.org/10.1016/S1359-8368(02)00024-0

[6] Zhao, C., Nie, H.C., Guo, X. and Guan, Z.D. (2014) Compression Performance of Composite Stiffened Panel after Stiffener Repair. Journal of Beijing University of Aeronautics and Astronautics, 40, 834-838.

[7] Chen, X.M., Zhang, A.Y., Chai, Y.N., Liu, H.W., Wang, L.L. and Yu, F. (2017) Investigation of Repair Technique for Aircraft Composite Wing Panel with Discrete-Source Damage. Journal of Mechanical Strength, 39, 551-556. 